\title{
First applications of a formula for the error of finite sinc interpolation
}

\author{
Jean-Paul Berrut
}

Received: 29 April 2008 / Revised: 19 December 2008 / Published online: 28 February 2009

(C) Springer-Verlag 2009

\begin{abstract}
In former articles we have given a formula for the error committed when interpolating a several times differentiable function by the sinc interpolant on a fixed finite interval. In the present work we demonstrate the relevance of the formula through several applications: correction of the interpolant through the insertion of derivatives to increase its order of convergence, improvement of the barycentric formula, rational sinc interpolants (with and without replacement of the (usually unknown) derivatives with finite differences), convergence acceleration through extrapolation and improvement of one-sided interpolants.
\end{abstract}

Mathematics Subject Classification (2000) $\quad$ Primary 65D05 · 41A80;

Secondary $65 \mathrm{~B} 05 \cdot 65 \mathrm{~B} 15$

\section{Introduction}

This paper is devoted to the interpolation of a several times differentiable function between equidistant nodes of a fixed finite interval. To be specific, let $f \in$ $C^{2 K+1}[-X, X]$ with $f^{(2 K+2)}$ absolutely integrable on $[-X, X], K \in \mathbb{N}, K \geq 2$. Assume that $f$ has been sampled at the equidistant points $x_{n}=n h, n=-N, \ldots, N$, $h=X / N$ and consider as in [7] the finite sinc interpolant

$$
C_{N}(f, h)(x)=\sum_{n=-N}^{N} " f_{n} \operatorname{sinc}\left(\frac{\pi}{h}\left(x-x_{n}\right)\right), \quad f_{n}:=f\left(x_{n}\right),
$$

Work partly supported by the Swiss National Science Foundation under grant Nr 200021-116122.

\section{J.-P. Berrut $(\varangle)$}

Department of Mathematics, University of Fribourg, 1700 Fribourg/Pérolles, Switzerland

e-mail: jean-paul.berrut@unifr.ch 
where the double prime signals that the first and last terms of the sum should be halved, and where the sinc function is given by

$$
\operatorname{sinc}(x):=\frac{\sin x}{x} .
$$

If $f$ is defined everywhere on $(-\infty, \infty)(1.1)$ is a truncation of its cardinal series. To such a function and to a interval length $h \in \mathbb{R}$, one indeed associates the sinc interpolant or cardinal series

$$
C(f, h)(x):=\sum_{n=-\infty}^{\infty} \operatorname{sinc}\left(\frac{\pi}{h}\left(x-x_{n}\right)\right) f_{n} .
$$

(1.2) is known to be an excellent approximant of $f$ if the latter is very smooth and decays rapidly enough as $|x| \rightarrow \infty$. For example, one has $C(f, h)(x)=f(x) \forall x$ when $f$ belongs to the Paley-Wiener class with exponent $\pi / h[19,21]$; also, $C(f, h)(x)$ converges exponentially toward $f(x)$ when $f$ is analytic in a symmetric strip about the real axis and decays fast enough on the lines that make up the boundary of the strip [19,21]. One obviously has [7]

$$
C_{N}(f, h)(x)=C\left(f^{*}, h\right)(x),
$$

where

$$
f^{*}(x)= \begin{cases}f(x), & |x|<X \\ f(x) / 2, & |x|=X \\ 0, & |x|>X\end{cases}
$$

$f^{*}$ usually has jumps at $x= \pm X$.

In the article [8], we have given the following formula for the error $C_{N}(f, h)-f$ on the interval.

Theorem 1.1 Let $f$ and $C_{N}(f, h)$ be defined as above. Then the difference $C_{N}(f, h)-$ $f$ is given by

$$
\begin{aligned}
& C_{N}(f, h)(x)=f(x)+\frac{(-1)^{N}}{2 \pi} \sin \left(\frac{\pi}{h} x\right) \sum_{k=1}^{K} b_{2 k}(x)(2 h)^{2 k}+\mathcal{O}\left(h^{2 K+2}\right), \\
& b_{2 k}(x):=2\left(1-4^{-k}\right) \frac{B_{2 k}}{(2 k) !}\left[\left(\frac{f(y)}{x-y}\right)^{(2 k-1)}(X)-\left(\frac{f(y)}{x-y}\right)^{(2 k-1)}(-X)\right],
\end{aligned}
$$

where the derivatives are taken with respect to $y$.

One may write $\sum b_{2 k}(x) h^{2 k}$ and replace $1-4^{-k}$ with $4^{k}-1$ in $b_{2 k}$, but the above notation will have advantages, e.g., for Theorem 7.1. In [8], we give a precise expression for the remainder term as well as the corresponding formula for an even number 
of interpolation points. The present paper discusses first uses of the formula which occurred to the author after its discovery.

Section 2 demonstrates the most obvious use of the formula, namely the successive subtraction from $C_{N}$ of the terms corresponding to increasing powers of $h$ to accelerate convergence. A formula for the derivatives of the Cauchy kernel as a function of those of $f$ greatly simplifies the process. In view of the singularity of the kernel, the formula is merely efficient far enough from the extremities $X$ and $-X$. In Sect. 3, we precise this fact by deriving that the evaluation point should not be closer to $\pm X$ than half the steplength.

Section 4 improves upon the barycentric formula for the full sinc interpolant through the application of (1.3) to accelerate the convergence of the denominator toward 1 . In Sect. 5, we extend this division process to finite interpolants. The result is impressive, with a rest error close to machine precision with about 50 points for Runge's function and a complete elimination of the distance restriction to the extremities and of the Gibbs phenomenon. Section 6 treats the realistic case in which no formula for the derivatives of $f$ at $\pm X$ is known and divided differences are used for their approximation. The results are perfect with centered differences while one-sided differences, as they are based on polynomial interpolation between equidistant points, are unstable for high orders, as expected. This leads to the conclusion that, whenever possible, some values of the sample should be sacrified at the extremities when using (1.3) to improve the approximation.

A formula such as (1.3) cries out for extrapolation. Section 7 gives the corresponding formulae; their application in the example of a steep function yields machine precision in the immediate vicinity of the extremities with 513 sample points. A last section on one-sided interpolants gives the formula corresponding to (1.3) in that case and an example where its use again leads to an excellent approximation.

\section{Direct use of the formula for improving upon $C_{N}$}

The use of (1.3) that immediately comes to mind is the approximation of $f$ with an improved error of $\mathcal{O}\left(h^{2 K+2}\right)$ instead of $\mathcal{O}\left(h^{2}\right)$ by subtracting from $C_{N}(f, h)$ the term consisting of the sum times the sine. The first issue to address then is the computation of the derivatives of $f(y) /(x-y)$ at the extremities as functions of the derivatives of $f$, for which we assume here that formulae are known, a hypothesis which will be lifted in subsequent sections. A useful formula for these derivatives is given in the following lemma.

Lemma 2.1 Assume that $f$ is $\ell$ times differentiable at $y \neq x$. Then

$$
\left(\frac{f(y)}{x-y}\right)^{(\ell)}=\sum_{k=0}^{\ell} \frac{\ell !}{k !} \frac{f^{(k)}(y)}{(x-y)^{\ell+1-k}} .
$$

Proof By induction or with Leibniz' rule of differentiation. 
Table 1 Interpolation errors for $f(x)=\cos (x)+\sinh (5 x)$ on $[-1,1]$ with $N=100$

\begin{tabular}{lrrrrr}
\hline$x$ & \multicolumn{1}{l}{1} & \multicolumn{1}{l}{ } & \multicolumn{1}{l}{3} & \multicolumn{1}{l}{14} \\
\hline $5.2632 \mathrm{e}-02$ & $3.4790 \mathrm{e}-03$ & $-5.3608 \mathrm{e}-07$ & $1.0718 \mathrm{e}-10$ & $-2.5979 \mathrm{e}-14$ & $-3.3307 \mathrm{e}-15$ \\
$1.5789 \mathrm{e}-01$ & $2.9344 \mathrm{e}-03$ & $-4.4994 \mathrm{e}-07$ & $8.9756 \mathrm{e}-11$ & $5.1070 \mathrm{e}-14$ & $6.9944 \mathrm{e}-14$ \\
$2.6316 \mathrm{e}-01$ & $-4.0832 \mathrm{e}-03$ & $6.1953 \mathrm{e}-07$ & $-1.2248 \mathrm{e}-10$ & $4.6629 \mathrm{e}-14$ & $2.1760 \mathrm{e}-14$ \\
$3.6842 \mathrm{e}-01$ & $-2.3903 \mathrm{e}-03$ & $3.5625 \mathrm{e}-07$ & $-6.8813 \mathrm{e}-11$ & $-8.8818 \mathrm{e}-15$ & $-2.1316 \mathrm{e}-14$ \\
$4.7368 \mathrm{e}-01$ & $4.7508 \mathrm{e}-03$ & $-6.8413 \mathrm{e}-07$ & $1.1817 \mathrm{e}-10$ & $1.2434 \mathrm{e}-14$ & $9.7700 \mathrm{e}-15$ \\
$5.7895 \mathrm{e}-01$ & $1.7145 \mathrm{e}-03$ & $-2.2127 \mathrm{e}-07$ & $4.9631 \mathrm{e}-12$ & $1.3678 \mathrm{e}-13$ & $3.9080 \mathrm{e}-14$ \\
$6.8421 \mathrm{e}-01$ & $-4.7646 \mathrm{e}-03$ & $1.9285 \mathrm{e}-07$ & $1.0892 \mathrm{e}-09$ & $-5.2616 \mathrm{e}-12$ & $-7.1054 \mathrm{e}-15$ \\
$7.8947 \mathrm{e}-01$ & $-3.3723 \mathrm{e}-04$ & $-7.3733 \mathrm{e}-07$ & $3.9360 \mathrm{e}-09$ & $-3.7744 \mathrm{e}-11$ & 0 \\
$8.9474 \mathrm{e}-01$ & $-2.4071 \mathrm{e}-02$ & $1.4016 \mathrm{e}-04$ & $-2.4982 \mathrm{e}-06$ & $9.3449 \mathrm{e}-08$ & $4.9738 \mathrm{e}-14$ \\
\hline
\end{tabular}

Inserted into (1.3), the formula shows that when $f \in C^{\infty}[-X, X]$ and all derivatives at the extremities vanish, the convergence is spectral, i.e., faster than any power of $h$. If $X$ is large enough that they are all smaller than, say, machine precision $v$, then that holds in practice too. We will concentrate here on examples for which that is not the case.

Example 1 We started with an entire function whose derivatives of all orders are very simple, $f(x)=\cos (x)+\sinh (5 x)$, and approximated $f$ on the interval $[-1,1]$. We interpolated with $2 N+1=201$ nodes $(h=0.01)$ and evaluated all functions at 20 equidistant points, with none of them a node.

Table 1 contains numerical values: the first column displays $x$, the second the error $C_{N}(f, h)(x)-f(x)$, the third the error with $C_{N}(f, h)$ corrected with the first term of the sum in (1.3), i.e., $C_{N}(f, h)(x)-\left(\sin ((\pi / h) x) b_{2}(x)(2 h)^{2}\right) / 2 \pi-f(x)$, and the remaining columns the error with the number $K$ of terms given as the column heading. We give the results for the $x$ on one side of 0 only since those on the other side lie qualitatively very close by.

The numbers show that the formula is very efficient with this relatively high number of nodes and they confirm that the error decreases like the even powers of $\widetilde{h}:=2 h-$ despite the presence of the sine factor containing $h$. They also reveal that the convergence is the slower the closer one comes to the extremities.

This behaviour becomes more dramatic if we decrease $N$ to 25 , see Table 2: we still have the decay as the powers of $\widetilde{h}^{2}$ in the center, while the improvement is much slower close to the endpoints, if there is one at all. The reason for this diverse behavior is the denominator present in the derivatives, recall Lemma 2.1. Not a problem when $x$ is far from the extremities, it becomes very small close to them, pushing the derivatives of $f(y) /(x-y)$ to larger and larger values as their order grows. If $h$ is not small enough, the increase in the derivatives may largely overcompensate the decay in the powers of $\widetilde{h}$, in which case the last term of the sum and the remainder term in (1.3) dominate all others, making it impossible to extract $f(x)$. This effect is even reinforced by the Bernoulli numbers $B_{2 k}$, which are at most 1 up to $k=6$, but then 
Table 2 Interpolation errors for $f(x)=\cos (x)+\sinh (5 x)$ on $[-1,1]$ with $N=25$

\begin{tabular}{lrrrrr}
\hline$x$ & \multicolumn{1}{l}{1} & \multicolumn{1}{l}{ } & \multicolumn{1}{l}{3} & \multicolumn{1}{l}{14} \\
\hline $5.2632 \mathrm{e}-02$ & $-6.3193 \mathrm{e}-02$ & $1.5569 \mathrm{e}-04$ & $-4.9800 \mathrm{e}-07$ & $1.6833 \mathrm{e}-09$ & $-5.9952 \mathrm{e}-15$ \\
$1.5789 \mathrm{e}-01$ & $-1.2553 \mathrm{e}-02$ & $3.0775 \mathrm{e}-05$ & $-9.8133 \mathrm{e}-08$ & $3.2991 \mathrm{e}-10$ & $9.3259 \mathrm{e}-15$ \\
$2.6316 \mathrm{e}-01$ & $7.5479 \mathrm{e}-02$ & $-1.8311 \mathrm{e}-04$ & $5.7923 \mathrm{e}-07$ & $-1.9047 \mathrm{e}-09$ & $-3.5527 \mathrm{e}-15$ \\
$3.6842 \mathrm{e}-01$ & $-4.9246 \mathrm{e}-02$ & $1.1736 \mathrm{e}-04$ & $-3.6265 \mathrm{e}-07$ & $1.0499 \mathrm{e}-09$ & $-1.5987 \mathrm{e}-14$ \\
$4.7368 \mathrm{e}-01$ & $-3.9421 \mathrm{e}-02$ & $9.0786 \mathrm{e}-05$ & $-2.5162 \mathrm{e}-07$ & $-7.6173 \mathrm{e}-11$ & $8.8818 \mathrm{e}-15$ \\
$5.7895 \mathrm{e}-01$ & $8.4033 \mathrm{e}-02$ & $-1.7378 \mathrm{e}-04$ & $7.9275 \mathrm{e}-08$ & $1.8601 \mathrm{e}-08$ & $-5.3291 \mathrm{e}-15$ \\
$6.8421 \mathrm{e}-01$ & $-2.5517 \mathrm{e}-02$ & $1.7840 \mathrm{e}-05$ & $1.3970 \mathrm{e}-06$ & $-1.0447 \mathrm{e}-07$ & $1.7906 \mathrm{e}-11$ \\
$7.8947 \mathrm{e}-01$ & $-2.4850 \mathrm{e}-02$ & $-7.8455 \mathrm{e}-04$ & $6.3689 \mathrm{e}-05$ & $-9.0653 \mathrm{e}-06$ & $7.5776 \mathrm{e}-06$ \\
$8.9474 \mathrm{e}-01$ & $-3.2892 \mathrm{e}-01$ & $2.7046 \mathrm{e}-02$ & $-6.5139 \mathrm{e}-03$ & $3.2409 \mathrm{e}-03$ & $-5.3035 \mathrm{e}+03$ \\
\hline
\end{tabular}

start growing rapidly, asymptotically as $\left|B_{2 k}\right| \sim 2 \cdot(2 \pi)^{-2 k} \cdot(2 k)$ ! [3, p. 38; 20, p. 380].

Remark In the genuine case where none of the derivatives of $f$ at $X$ or $-X$ vanishes, the algorithm given in [15] might be more efficient than (2.1). This would be only marginal, though, and speed is not our concern here.

It should be noted that all the corrected approximants calculated above interpolate in the $x_{n}$ for, at the nodes, the sine factor annihilates the influence of the sum in (1.3).

\section{A relation between the sampling interval $h$ and the evaluation point $x$}

In order to avoid the effect mentioned at the end of the preceding section, namely that the last terms of our formula dominate $f(x)$, it is natural to require that the terms at least do not grow with $k$. The factor $1-4^{-k}$ in $b_{2 k}(x)$ indeed grows, but is bounded by 1 , while the sequence of the $B_{2 k}$ first decreases, but then increases very rapidly. In order for the error to become small with a reasonable number of terms, $x$ should be far enough from the extremities for the factors

$$
c_{2 k}(x, h):=\frac{1}{(2 k) !}\left[\left(\frac{f(y)}{x-y}\right)^{(2 k-1)}(X)-\left(\frac{f(y)}{x-y}\right)^{(2 k-1)}(-X)\right](2 h)^{2 k}
$$

to decrease with $k$. To determine how far $x$ should stay away from the endpoints, let us assume that it comes close to $X$. Then the first term in the brackets will become much larger than the second and, with Lemma 2.1, (3.1) will be about

$$
\begin{aligned}
c_{2 k}(x, h) \approx & \frac{(2 h)^{2 k}}{(2 k) !} \sum_{j=0}^{2 k-1} \frac{(2 k-1) !}{j !} \frac{f^{(j)}(X)}{(x-X)^{2 k-1+1-j}} \\
& =\frac{1}{2 k}\left(\frac{2 h}{x-X}\right)^{2 k} \sum_{j=0}^{2 k-1} \frac{f^{(j)}(X)}{j !}(x-X)^{j}
\end{aligned}
$$


The sum on the right is the head of a Taylor sum of $f$ at $x$ about $X$; if $f \in C^{\infty}[-X, X]$ it will approach $f(x)$ with increasing $k$, rapidly as we assume that $x \approx X$, and

$$
c_{2 k}(x, h) \approx \frac{1}{2 k}\left(\frac{2 h}{X-x}\right)^{2 k} f(x) .
$$

Since the factor $1 / k$ ensures decay, a sufficient condition for the decrease of the $c_{2 k}$ is $h \leq \frac{X-x}{2}$ : the interval length $h$ should be at most half the distance from $x$ to the boundary.

\section{Correction of the barycentric formula}

To evaluate the (full) sinc interpolant (1.2), one will have to truncate the series, for example by replacing it with $C_{N}(f, h)(x)$, which in turn requires the calculation of $2 N+1$ sines. This may be avoided by writing $C(f, h)$ in the alternate form $[5,18,23]$

$$
C(f, h)(x)=\frac{h}{\pi} \sin \frac{\pi}{h} x \sum_{n=-\infty}^{\infty}(-1)^{n} \frac{f_{n}}{x-x_{n}}
$$

and similarly for $C_{N}$. Both may then be evaluated using only one sine. However, these formulae may be unstable in the vicinity of large multiples of $h$ [5]. This flaw has been recently fixed by Gautschi [16] who replaces the factor $\sin \frac{\pi}{h} x$ in front of the sum by $\sin \frac{\pi}{h}\left(x-x_{0}\right)$, with $x_{0}$ the node closest to $x$, and modifies the terms of the sum accordingly. This works perfectly well, but requires the determination of $x_{0}$ for every evaluation point $x$.

In [5], we have suggested to eliminate the unstable factor by dividing $C(f, h)$ by $1 \equiv C(1, h)$, both written as in (4.1), to obtain the barycentric formula

$$
C(f, h)(x)=\frac{\sum_{n=-\infty}^{\infty} \frac{(-1)^{n}}{x-x_{n}} f_{n}}{\sum_{n=-\infty}^{\infty} \frac{(-1)^{n}}{x-x_{n}}}
$$

(The reader may consult [10] for a recent discussion of such formulae which puts (4.2) into a wider context.) Proceeding in the same way for $C_{N}$ yields the rational interpolant

$$
R(x)=\sum_{n=-N}^{N}{ }^{\prime \prime} \frac{(-1)^{n}}{x-x_{n}} f_{n} / \sum_{n=-N}^{N}{ }^{\prime \prime} \frac{(-1)^{n}}{x-x_{n}}
$$

suggested in [4] for arbitrary sets of interpolation nodes $\left\{x_{k}\right\} . R$ has since had several applications, e.g., for the exponentially fast converging approximation of functions given at conformally shifted Chebyshev points [2] and its use in the solution of differential equations $[1,22]$. For the equidistant nodes considered here, it has been 
conjectured in [4] and proved recently in the much more general paper [12] that its convergence is $\mathcal{O}\left(h^{2}\right)$ even when $f$ is very smooth.

The big drawback of (4.2) is that the terms in its denominator decrease merely as $1 /\left(x-x_{n}\right)$ as $n \rightarrow \infty$, so that a precise computation of the sum requires an extremely large number of them. To avoid this difficulty, we have suggested in [5] to divide (4.1) by the corresponding interpolant of a fast decreasing function (and to multiply the quotient again by the same function or to premultiply $f$ with it), which led to speedups of up to a factor of 10 in comparison with the direct evaluation of (4.1). The drawback of this approach is that the function used to construct the barycentric formula depends on $f$.

Our formula (1.3) for the finite sinc interpolant offers another approach: we may cut the sinc interpolant of the denominator 1 after a finite number of terms (about the same number as considered in the evaluation of $C_{N}$, see below) and correct the error with the formula to get an approximation of 1 with machine precision.

We assume in this section that $f$ decreases fast enough so that (1.1) gives $C(f, h)$ with the desired accuracy and write $[-X, X]$ for the corresponding interval, $X=N h$. To avoid the problem at the extremities studied in Sects. 2 and 3, we will enlarge the interval from $[-X, X]$ to $[-Z, Z], Z=M h, M>N$. With Lemma 2.1 for $f \equiv 1$, and neglecting the $\mathcal{O}\left(h^{2 K+2}\right)$-term, formula (1.3) reads

$$
\begin{aligned}
& C_{M}(1, h)(x) \\
& \quad \approx 1+\frac{(-1)^{M}}{2 \pi} \sin \left(\frac{\pi}{h} x\right) \sum_{k=1}^{K}\left(1-4^{-k}\right) \frac{B_{2 k}}{k}\left[\frac{1}{(x-Z)^{2 k}}-\frac{1}{(x+Z)^{2 k}}\right](2 h)^{2 k}
\end{aligned}
$$

or with $C_{M}$ written as in (4.1)

$$
\begin{aligned}
1 & \approx \frac{h}{\pi} \sin \frac{\pi}{h} x\left[\sum_{n=-M}^{M}{ }^{\prime \prime} \frac{(-1)^{n}}{x-x_{n}}\right. \\
& \left.+(-1)^{M} \sum_{k=1}^{K}\left(1-4^{-k}\right) \frac{B_{2 k}}{k}\left(\frac{1}{(x+Z)^{2 k}}-\frac{1}{(x-Z)^{2 k}}\right)(2 h)^{2 k-1}\right] .
\end{aligned}
$$

Dividing $C_{N}$ written as in (4.1) by (4.4) and cancelling the common factor $\frac{h}{\pi} \sin \frac{\pi}{h} x$ we obtain the following approximation for $C(f, h)(x)$ :

$$
\begin{aligned}
& C_{N, M}(f, h)(x) \\
& :=\frac{\sum_{n=-N}^{N} \frac{(-1)^{n}}{x-x_{n}} f_{n}}{\sum_{n=-M}^{M} \frac{(-1)^{n}}{x-x_{n}}+(-1)^{M} \sum_{k=1}^{K}\left(1-4^{-k}\right) \frac{B_{2 k}}{k}\left(\frac{1}{(x+Z)^{2 k}}-\frac{1}{(x-Z)^{2 k}}\right)(2 h)^{2 k-1}} .
\end{aligned}
$$

Note that this formula does not contain any derivative. 
It remains to determine $Z$ or, equivalently, $M$. In the same spirit as in the preceding section, we shall first assume that $K$ is small enough for $\frac{B_{2 k}}{k}\left(1-4^{-k}\right)<1 \forall k \leq$ $K+1$-which holds up to $K=7$-find a condition on $k$ such that $h$ times the remaining factors in the terms of the $k$-sum is less than machine precision $v$ and so derive a condition for the minimal distance $(M-N) h$ from $x$ to the boundary. (The factor $h$ comes from the fact that numerator and denominator have been multiplied by $\frac{\pi}{h}$ and for $h<\pi$ are therefore that much too large in comparison with the computed sinc interpolant.) If $N$ is large enough, and since $x \in[-X, X]$, there holds $|x \pm Z|>Z-X=(M-N) h$ so that we want $h \frac{2}{(Z-X)^{2(K+1)}}(2 h)^{2 K+1}<v$ or

$$
M-N>2 \cdot \sqrt[2(K+1)]{1 / v}
$$

To minimize this number, one should take the largest possible $K, K=7$, which leads to $M=N+20$ for the usual double precision of $v=10^{-16}$. (This application is the only one in this paper where we impose such a limitation upon $K$.) As a result of the crude approximation $\left|(x+Z)^{-2(K+1)}-(x-Z)^{-2(K+1)}\right|<2(Z-X)^{-2(K+1)}$ and the factor $\sin \frac{\pi}{h} x$, a significantly smaller $M$ suffices in practice.

Example 2 We have tested the rapidly decreasing function $f(x)=e^{-x^{2}}[\cos x+$ $\sinh x]$ on the interval $[-7,7]$ (which contains its numerical support) and evaluated its sinc interpolant in points approaching the node $b=0.7$, namely $x=b-d$ for $d=5 \times 10^{-2}, 10^{-2}, 10^{-5}, 10^{-10}, 10^{-14}$. We took 21 points on the interval $[-7,7]$, added $M-N=10$ points on each side for the approximation of the function 1 and corrected the error with $K=7$ terms. In Table 3 the first block gives the results with the classical definition (1.1) of the interpolant, the second with the finite formula corresponding to (4.1) and the last with (4.5). The first column of every block gives the evaluation abscissa $x$, the second the computed value $C_{10}(f, h)(x)$, resp. $C_{10,20}(f, h)(x)$, the third the true value $f(x)$ and the last the error (without its sign, which is everywhere positive but for the last value). Comparing the errors for the second block with those obtained with the first formula documents the hard time of the cheap formula in the vicinity of the node, whereas the values obtained with (4.5) are practically identical to those computed with (1.1).

It should be mentioned that if one is willing to use an "if"-statement, then one may simply take $M=N$ whenever $x$ is not near one of the endpoints $\pm Z$. Note finally that $C_{N, M}$ is just a generalization of $R$ in (4.3): letting $M=N$ and $K=0$ recovers the latter.

\section{Quotient of corrected sinc interpolants}

The above assumed that $f$ decreased so rapidly that it was zero close to the extremities and $x$ therefore never close enough to $\pm X$ for the terms of the sum in (1.3) to increase. Suppose now that this is not the case, that $x$ is so close to $X$ or $-X$ that the condition given in Sect. 3 is not satisfied and the terms in (1.3) grow rapidly. Then the corrected 
Table 3 Interpolation errors for $e^{-x^{2}}[\cos x+\sinh x]$ on $[-7,7]$ with $N=10$

\begin{tabular}{clll}
\hline$x$ & Approximation & $f(x)$ & Absolute error \\
\hline Original sinc formula & & & \\
0.6499999999999999 & 0.9787308391072040 & 0.9784109868473423 & $3.198522598617526 \mathrm{e}-04$ \\
0.6899999999999999 & 0.9426325359376126 & 0.9425586041299581 & $7.393180765458141 \mathrm{e}-05$ \\
0.6999900000000000 & 0.9333003087102524 & 0.9333002324304758 & $7.627977660629170 \mathrm{e}-08$ \\
0.6999999998999999 & 0.9332909093552330 & 0.9332909093544698 & $7.631673071273326 \mathrm{e}-13$ \\
0.6999999999999900 & 0.9332909092612472 & 0.9332909092612469 & $3.330669073875470 \mathrm{e}-16$ \\
Cheap formula (4.1) & & & \\
0.6499999999999999 & 0.9787308391072180 & 0.9784109868473423 & $3.198522598757414 \mathrm{e}-04$ \\
0.6899999999999999 & 0.9426325359376866 & 0.9425586041299581 & $7.393180772852226 \mathrm{e}-05$ \\
0.6999900000000000 & 0.9333003087820606 & 0.9333002324304758 & $7.635158483232374 \mathrm{e}-08$ \\
0.6999999998999999 & 0.9332978642423857 & 0.9332909093544698 & $6.954887915910923 \mathrm{e}-06$ \\
0.6999999999999900 & 1.002885713349400 & 0.9332909092612469 & $6.959480408815311 \mathrm{e}-02$ \\
Corrected barycentric formula & & & \\
0.6499999999999999 & 0.9787308391072037 & 0.9784109868473423 & $3.198522598614195 \mathrm{e}-04$ \\
0.6899999999999999 & 0.9426325359376129 & 0.9425586041299581 & $7.393180765480345 \mathrm{e}-05$ \\
0.6999900000000000 & 0.9333003087102519 & 0.9333002324304758 & $7.627977616220250 \mathrm{e}-08$ \\
0.6999999998999999 & 0.9332909093552328 & 0.9332909093544698 & $7.630562848248701 \mathrm{e}-13$ \\
0.6999999999999900 & 0.9332909092612465 & 0.9332909092612469 & $4.440892098500626 \mathrm{e}-16$ \\
\hline
\end{tabular}

sinc interpolant

$$
C_{N}(f, h)(x)-\frac{(-1)^{N}}{2 \pi} \sin \left(\frac{\pi}{h} x\right) \sum_{k=1}^{K} b_{2 k}(x)(2 h)^{2 k}
$$

will be about

$$
-\frac{(-1)^{N}}{2 \pi} \sin \left(\frac{\pi}{h} x\right) b_{2 K}(x)(2 h)^{2 K}
$$

an expression without obvious connection to $f(x)$. On closer inspection, and using the same Taylor series argument as in Sect. 3, one however realizes that, as $x$ comes close to $X,(5.2)$ approaches

$$
-\frac{(-1)^{N}}{2 \pi} 2\left(1-4^{-k}\right) \frac{B_{2 K}}{2 K} \sin \left(\frac{\pi}{h} x\right)\left(\frac{2 h}{X-x}\right)^{2 K} f(x) ;
$$

for a given $x$ this approximation to (5.1) is the better, the larger $K$ (which determines the number of terms in the Taylor series). This leads quite naturally to recover $f(x)$ close to the extremities by dividing the corrected sinc interpolant to $f$ by the interpolant with the same number of correcting terms for a known function, which 
Table 4 Errors with the quotient of corrected sinc interpolants for $f(x)=\cos (x)+\sinh (5 x)$ on $[-1,1]$ with $N=25$

\begin{tabular}{lrrrrr}
\hline$x$ & \multicolumn{1}{l}{1} & \multicolumn{1}{l}{ 2 } & \multicolumn{1}{l}{14} \\
\hline $5.2632 \mathrm{e}-02$ & $-6.3193 \mathrm{e}-02$ & $1.5574 \mathrm{e}-04$ & $-4.9822 \mathrm{e}-07$ & $1.6853 \mathrm{e}-09$ & $-6.2172 \mathrm{e}-15$ \\
$1.5789 \mathrm{e}-01$ & $-1.2553 \mathrm{e}-02$ & $3.0819 \mathrm{e}-05$ & $-9.8369 \mathrm{e}-08$ & $3.3234 \mathrm{e}-10$ & $2.2204 \mathrm{e}-16$ \\
$2.6316 \mathrm{e}-01$ & $7.5479 \mathrm{e}-02$ & $-1.8390 \mathrm{e}-04$ & $5.8428 \mathrm{e}-07$ & $-1.9691 \mathrm{e}-09$ & $-1.7764 \mathrm{e}-15$ \\
$3.6842 \mathrm{e}-01$ & $-4.9246 \mathrm{e}-02$ & $1.1885 \mathrm{e}-04$ & $-3.7496 \mathrm{e}-07$ & $1.2589 \mathrm{e}-09$ & $-4.4409 \mathrm{e}-15$ \\
$4.7368 \mathrm{e}-01$ & $-3.9421 \mathrm{e}-02$ & $9.4585 \mathrm{e}-05$ & $-2.9559 \mathrm{e}-07$ & $9.8745 \mathrm{e}-10$ & $1.4211 \mathrm{e}-14$ \\
$5.7895 \mathrm{e}-01$ & $8.4033 \mathrm{e}-02$ & $-2.0488 \mathrm{e}-04$ & $6.3252 \mathrm{e}-07$ & $-2.0994 \mathrm{e}-09$ & $-1.7764 \mathrm{e}-15$ \\
$6.8421 \mathrm{e}-01$ & $-2.5517 \mathrm{e}-02$ & $6.9575 \mathrm{e}-05$ & $-2.1160 \mathrm{e}-07$ & $6.9677 \mathrm{e}-10$ & $-3.5527 \mathrm{e}-14$ \\
$7.8947 \mathrm{e}-01$ & $-2.4850 \mathrm{e}-02$ & $1.6563 \mathrm{e}-04$ & $-4.9471 \mathrm{e}-07$ & $1.6138 \mathrm{e}-09$ & $1.7764 \mathrm{e}-14$ \\
$8.9474 \mathrm{e}-01$ & $-3.2892 \mathrm{e}-01$ & $-2.1830 \mathrm{e}-04$ & $6.3873 \mathrm{e}-07$ & $-2.0601 \mathrm{e}-09$ & $-7.1054 \mathrm{e}-15$ \\
\hline
\end{tabular}

we naturally take as the constant 1 . We therefore suggest to approximate $f$ by the following interpolant:

$$
Q_{N, K}(f, h)(x):=\frac{C_{N}(f, h)(x)-\frac{(-1)^{N}}{2 \pi} \sin \left(\frac{\pi}{h} x\right) \sum_{k=1}^{K} b_{2 k}(x)(2 h)^{2 k}}{C_{N}(1, h)(x)-\frac{(-1)^{N}}{2 \pi} \sin \left(\frac{\pi}{h} x\right) \sum_{k=1}^{K} d_{2 k}(x)(2 h)^{2 k}},
$$

where the $d_{2 k}$ denote the $b_{2 k}$ of the function 1. After inserting (4.1) for $C_{N}$ and cancelling the common factor $\frac{h}{\pi} \sin \left(\frac{\pi}{h} x\right)$, this becomes

$$
\begin{aligned}
& Q_{N, K}(f, h)(x) \\
& =\frac{\sum_{n=-N}^{N} \frac{(-1)^{n}}{x-x_{n}} f_{n}-(-1)^{N} \sum_{k=1}^{K} b_{2 k}(x)(2 h)^{2 k-1}}{\sum_{n=-N}^{N}{ }^{\prime \prime} \frac{(-1)^{n}}{x-x_{n}}+(-1)^{N} \sum_{k=1}^{K}\left(1-4^{-k}\right) \frac{B_{2 k}}{k}\left(\frac{1}{(x+X)^{2 k}}-\frac{1}{(x-X)^{2 k}}\right)(2 h)^{2 k-1}} .
\end{aligned}
$$

Notice that the division cancels the factors $1 / 2$ at the extremities and gives the values $f( \pm X)$ back.

Example 3 We have re-computed Example 1 with $N=25$ that led to Table 2. As displayed in Table 4, the interpolation is now accurate up to machine precision and this on the whole interval, not only far away from and very close to the extremities. The interpolation scheme is still perfectly stable and with $N=100$ machine precision is already attained with $K=4$. Note also the regularity of the error along the whole interval. (We might add that in one example the maximal error was midway between 0 and $\pm X$; more tests are needed here).

In fact, the interpolant (5.3) is much better than might be expected from a separate look at its numerator and denominator: with $K=14$ one might decrease $N$ to 8 before leaving the machine precision regime! Why this? In contrast to a division 
Table 5 Same as Table 4 but for $f(x)=x+1 /\left(1+25 x^{2}\right)$ and $N=30$

\begin{tabular}{lrrrrr}
\hline$x$ & \multicolumn{1}{l}{1} & \multicolumn{1}{l}{ } & \multicolumn{1}{l}{14} \\
\hline $5.2632 \mathrm{e}-02$ & $-2.3206 \mathrm{e}-06$ & $-1.8452 \mathrm{e}-08$ & $-3.0648 \mathrm{e}-09$ & $-3.1077 \mathrm{e}-09$ & $-3.1075 \mathrm{e}-09$ \\
$1.5789 \mathrm{e}-01$ & $1.0031 \mathrm{e}-05$ & $2.7674 \mathrm{e}-08$ & $4.5965 \mathrm{e}-09$ & $4.6608 \mathrm{e}-09$ & $4.6605 \mathrm{e}-09$ \\
$2.6316 \mathrm{e}-01$ & $-1.1705 \mathrm{e}-05$ & $-1.2098 \mathrm{e}-08$ & $-2.0094 \mathrm{e}-09$ & $-2.0375 \mathrm{e}-09$ & $-2.0374 \mathrm{e}-09$ \\
$3.6842 \mathrm{e}-01$ & $-1.2577 \mathrm{e}-05$ & $-5.3376 \mathrm{e}-09$ & $-8.8654 \mathrm{e}-10$ & $-8.9895 \mathrm{e}-10$ & $-8.9889 \mathrm{e}-10$ \\
$4.7368 \mathrm{e}-01$ & $9.2284 \mathrm{e}-05$ & $1.7023 \mathrm{e}-08$ & $2.8274 \mathrm{e}-09$ & $2.8669 \mathrm{e}-09$ & $2.8667 \mathrm{e}-09$ \\
$5.7895 \mathrm{e}-01$ & $-2.7143 \mathrm{e}-04$ & $-2.1859 \mathrm{e}-08$ & $-3.6306 \mathrm{e}-09$ & $-3.6814 \mathrm{e}-09$ & $-3.6812 \mathrm{e}-09$ \\
$6.8421 \mathrm{e}-01$ & $6.2924 \mathrm{e}-04$ & $2.0757 \mathrm{e}-08$ & $3.4476 \mathrm{e}-09$ & $3.4958 \mathrm{e}-09$ & $3.4956 \mathrm{e}-09$ \\
$7.8947 \mathrm{e}-01$ & $-1.3696 \mathrm{e}-03$ & $-1.5413 \mathrm{e}-08$ & $-2.5601 \mathrm{e}-09$ & $-2.5959 \mathrm{e}-09$ & $-2.5957 \mathrm{e}-09$ \\
$8.9474 \mathrm{e}-01$ & $3.4083 \mathrm{e}-03$ & $7.8380 \mathrm{e}-09$ & $1.3016 \mathrm{e}-09$ & $1.3198 \mathrm{e}-09$ & $9.6871 \mathrm{e}-10$ \\
\hline
\end{tabular}

such as that used in Sect. 4, which divided by 1 computed as accurately as possible in $[-X, X]$, here we divided two functions that are imprecise close to the extremities. There, intuitively, the interpolants to all functions have the tendency to oscillate in the same (multiplicative) manner between the nodes and their quotient eliminates this common multiplicative factor.

Remark Gibbs' phenomenon is another instance of a multiplicative phenomenon. Dividing by the approximation of the same complexity of a known function often practically eliminates it, as we will document in [9]. The step from the approximation (1.1) to (4.5) and its generalization in the passage from (5.1) to (5.3) are examples of such an alleviation of the phenomenon.

Example 4 The function used up to this point is entire. We have also experimented with the meromorphic $f(x)=x+1 /\left(1+a^{2} x^{2}\right)$ and have let the poles $\pm i / a$ come down from infinity along the imaginary axis by increasing $a$, still interpolating on the interval $[-1,1]$. We have evaluated the derivatives with MATLAB's symbolic toolbox. With $a=1$, we retain machine precision with $N=11$ ( 23 points), whereas $a=5$ (asymmetric Runge's example) requires about $N=50$. It is instructive to study the numbers when such a high precision is not attained. In Table 5 we give the same numbers as in Table 4 for the asymmetric Runge function and $N=30$. As above, the plain sinc interpolant is much better in the center of the interval than close to its boundary. The first correcting term improves the approximation to a very regular one and brings a gain of five orders of magnitude and the second term brings another order. Then the procedure gets stuck, extra terms do not bring much improvement.

\section{Fully discrete interpolation}

Except in special cases such as the barycentric formula (Sect. 4), the correction methods presented in earlier sections require knowledge of the precise (continuous) 
derivatives of $f$ at the extremities, which evidently greatly limits their applicability. Automatic differentiation will be of great help here. Another obvious cure to this drawback is to (try to) replace the derivatives in the coefficients $b_{2 k}(x)$ with finite differences (FD) of sufficiently high order to essentially obtain the same accuracy. Finite differences are especially suited here since $f$ is sampled at equispaced points. One should thereby distinguish two cases: the genuine one occurs when one wishes to approximate up to the boundary and no extra value may be obtained outside $[-X, X]$, the other where one does not need to approximate up to the boundary and the last values at both extremities may be used to compute derivatives.

In the first case one will replace the derivatives with one-sided FD. We computed their weights as well as those of the centered formulae used below with an algorithm by Fornberg, who notes in [13, p. 19], that "the best polynomial-based approximation to $d^{k} f / d x^{k}$ at some point $\xi$ is obtained by differentiating Lagrange's interpolating polynomial". Fornberg's algorithms have the advantage that the same program calculates the weights for the derivatives at any gridpoint, in particular one-sided as well as centered FD. We have coded in MATLAB the procedure given on page 168 of his book. (Fornberg has proposed another method of calculating the weights in [14]).

As merely even powers of $h$ arise in (1.3) it is natural to aim at a global error $\mathcal{O}\left(h^{(2 K+2)}\right)$. In (1.3) the derivative $\left(\frac{f(y)}{x-y}\right)^{(2 k-1)}$ is multiplied by $h^{2 k}$ and should therefore be calculated with an error $\mathcal{O}\left(h^{2(K-k+1)}\right)$ to warrant that global error. On the other hand, in the calculation of odd order derivatives for centered differences and of derivatives of any order for one-sided ones, the number of abscissae equals the sum of the orders of derivative and accuracy [13, p. 16-17], here $2 K+1$, a number independent of the order of the derivative! We have therefore evaluated all derivatives on the right of (2.1) with that number of points; our experiments have confirmed the efficiency of this choice. Note that for the left-hand derivative at the right extremity the coefficients must be used backwards and a negative sign must be added to odd order derivatives.

Example 5 We have again considered Example 1 with $N=25$ and reconstructed Table 4, this time using centered differences with $2 K+1$ points, which requires values of $f$ at abscissae outside of the interval of interpolation. All results are exactly the same as in Table 4 up to the last one of the last column (error $\mathcal{O}\left(h^{30}\right)$ ) which is about $10^{-11}$.

The use of one-sided differences to avoid abscissae outside $[-1,1]$ is somewhat less convincing. Table 5 (asymmetric Runge function) is practically duplicated, up to the last entry which worsens to $1.9 \times 10^{-6}$. But, as documented in Table 6 (steep entire function) constructed with the same data as Table 4, while the elimination of the first terms is always very efficient, the computation of the derivatives with onesided differences will often degrade to become useless: such approximations amount to computing the derivatives of polynomial interpolants at the endpoints of the interval of interpolation, and those become severely ill-conditioned if not divergent with a growing number of equidistant nodes. 
Table 6 Same as Table 4, but with derivatives approximated by one-sided FD

\begin{tabular}{lrrrrr}
\hline$x$ & \multicolumn{1}{l}{1} & \multicolumn{1}{l}{ } & \multicolumn{1}{l}{3} & \multicolumn{1}{l}{14} \\
\hline $5.2632 \mathrm{e}-02$ & $-6.3193 \mathrm{e}-02$ & $1.5575 \mathrm{e}-04$ & $-5.7002 \mathrm{e}-07$ & $-4.2129 \mathrm{e}-08$ & $-4.1991 \mathrm{e}-08$ \\
$1.5789 \mathrm{e}-01$ & $-1.2553 \mathrm{e}-02$ & $3.0821 \mathrm{e}-05$ & $-1.1282 \mathrm{e}-07$ & $-9.0797 \mathrm{e}-09$ & $-8.5281 \mathrm{e}-09$ \\
$2.6316 \mathrm{e}-01$ & $7.5479 \mathrm{e}-02$ & $-1.8391 \mathrm{e}-04$ & $6.7358 \mathrm{e}-07$ & $5.9787 \mathrm{e}-08$ & $5.2997 \mathrm{e}-08$ \\
$3.6842 \mathrm{e}-01$ & $-4.9246 \mathrm{e}-02$ & $1.1885 \mathrm{e}-04$ & $-4.3606 \mathrm{e}-07$ & $-4.3361 \mathrm{e}-08$ & $-3.6299 \mathrm{e}-08$ \\
$4.7368 \mathrm{e}-01$ & $-3.9421 \mathrm{e}-02$ & $9.4590 \mathrm{e}-05$ & $-3.4856 \mathrm{e}-07$ & $-3.9627 \mathrm{e}-08$ & $-3.1322 \mathrm{e}-08$ \\
$5.7895 \mathrm{e}-01$ & $8.4033 \mathrm{e}-02$ & $-2.0489 \mathrm{e}-04$ & $7.6269 \mathrm{e}-07$ & $1.0190 \mathrm{e}-07$ & $7.5983 \mathrm{e}-08$ \\
$6.8421 \mathrm{e}-01$ & $-2.5517 \mathrm{e}-02$ & $6.9579 \mathrm{e}-05$ & $-2.6513 \mathrm{e}-07$ & $-4.3273 \mathrm{e}-08$ & $-3.0570 \mathrm{e}-08$ \\
$7.8947 \mathrm{e}-01$ & $-2.4850 \mathrm{e}-02$ & $1.6564 \mathrm{e}-04$ & $-6.6929 \mathrm{e}-07$ & $-1.4033 \mathrm{e}-07$ & $-5.8585 \mathrm{e}-07$ \\
$8.9474 \mathrm{e}-01$ & $-3.2892 \mathrm{e}-01$ & $-2.1832 \mathrm{e}-04$ & $1.0755 \mathrm{e}-06$ & $2.6490 \mathrm{e}-07$ & $-3.1523 \mathrm{e}-03$ \\
\hline
\end{tabular}

\section{Extrapolated sinc interpolation}

Improved sinc interpolants were obtained in Sect. 6 by subtracting from them the leading terms of our error formula (1.3) with the derivatives replaced by FD of sufficiently high order; this does not require knowledge of derivatives of $f$. While the results were excellent if one may abandon sample values at the extremities to use them in evaluating centered FD, the correction by one-sided FD was negatively influenced by ill-conditioning and by the Runge phenomenon inherent to the interpolation between equidistant points.

On the other hand, the very nature of (1.3) cries out for extrapolation along the lines of Romberg quadrature. We treat here only the case of the doubling of the number of nodes at each step. If we denote by $I:=\int_{0}^{X} f(x) d x$ an integral to be approximated and by $T^{(0)}(h):=h \sum_{k=0}^{N} f\left(x_{n}\right), x_{n}=n h, h=\frac{X}{N}$, its trapezoidal approximation as a function of $h$, i.e., of $N$, then the formula for the elements of the $\ell$ th column of the Romberg matrix reads

$$
\begin{aligned}
T^{(\ell)}\left(\frac{h}{2}\right):= & \frac{4^{\ell} T^{(\ell-1)}\left(\frac{h}{2}\right)-T^{(\ell-1)}(h)}{4^{\ell}-1} \\
= & I+\frac{1}{\prod_{i=1}^{\ell}\left(4^{i}-1\right)} \sum_{k=\ell+1}^{K}\left[\prod_{i=1}^{\ell}\left(4^{i}-4^{k}\right)\right] \\
& \times \frac{B_{2 k}}{(2 k) !}\left(\frac{h}{2}\right)^{2 k}+\mathcal{O}\left(h^{2 K+2}\right) .
\end{aligned}
$$

(see [17], where the $B_{2 q}$ seem to denote the absolute values of the usual Bernoulli numbers). By generalizing the steps leading to that formula, one obtains the corresponding one for the sinc interpolant as follows. 
Theorem 7.1 Let the same assumptions as in Theorem 1.1 be valid and for a given $x$ define $C^{(0)}(h):=C_{N}(f, h)(x)$. Then

$$
\begin{aligned}
C^{(1)}\left(\frac{h}{2}\right):= & \frac{4 C^{(0)}\left(\frac{h}{2}\right)-2(-1)^{N} \cos \left(\frac{\pi}{h} x\right) C^{(0)}(h)}{d^{(1)}(h)} \\
= & f(x)+\frac{1}{2 \pi d^{(1)}(h)} \sin \left(\frac{\pi}{h / 2} x\right) \sum_{k=2}^{K}\left[4-4^{k}\right] b_{2 k}(x) h^{2 k} \\
& +\mathcal{O}\left(h^{2 K+2}\right)
\end{aligned}
$$

where

$$
d^{(1)}(h)=4-2(-1)^{N} \cos \left(\frac{\pi}{h} x\right)
$$

and for $\ell=2, \ldots, K$

$$
\begin{aligned}
C^{(\ell)}\left(\frac{h}{2}\right):= & \frac{4^{\ell} C^{(\ell-1)}\left(\frac{h}{2}\right)-2 \cos \left(\frac{\pi}{h} x\right) \frac{d^{(\ell-1)}(2 h)}{d^{(\ell-1)}(h)} C^{(\ell-1)}(h)}{4^{\ell}-2 \cos \left(\frac{\pi}{h} x\right) \frac{d^{(\ell-1)}(2 h)}{d^{(\ell-1)}(h)}} \\
= & f(x)+\frac{1}{2 \pi} \frac{\sin \left(\frac{\pi}{h / 2} x\right)}{d^{(\ell)}(h)} \sum_{k=\ell+1}^{K}\left[\prod_{i=1}^{\ell}\left(4^{i}-4^{k}\right)\right] b_{2 k}(x) h^{2 k} \\
& +\mathcal{O}\left(h^{2 K+2}\right)
\end{aligned}
$$

with

$$
d^{(\ell)}(h)=d^{(\ell-1)}(h)\left[4^{\ell}-2 \cos \left(\frac{\pi}{h} x\right) \frac{d^{(\ell-1)}(2 h)}{d^{(\ell-1)}(h)}\right] .
$$

Proof By induction: one has from (1.3)

$$
C^{(0)}(h)=f(x)+\frac{(-1)^{N}}{2 \pi} \sin \left(\frac{\pi}{h} x\right) \sum_{k=1}^{K} b_{2 k}(x) 4^{k} h^{2 k}+\mathcal{O}\left(h^{2 K+2}\right),
$$

and

$$
C^{(0)}(h / 2)=f(x)+\frac{(-1)^{2 N}}{2 \pi} \sin \left(\frac{\pi}{h / 2} x\right) \sum_{k=1}^{K} b_{2 k}(x) h^{2 k}+\mathcal{O}\left(h^{2 K+2}\right) .
$$


Using $\sin \left(\frac{\pi}{h / 2} x\right)=2 \sin \left(\frac{\pi}{h} x\right) \cos \left(\frac{\pi}{h} x\right)$ in the second formula, one is led to consider

$$
\begin{aligned}
& 4 C^{(0)}\left(\frac{h}{2}\right)-(-1)^{N} 2 \cos \left(\frac{\pi}{h} x\right) C^{(0)}(h) \\
& =d^{(1)}(h) f(x)+\frac{1}{2 \pi} \sin \left(\frac{\pi}{h / 2} x\right) \sum_{k=2}^{K}\left[4-4^{k}\right] b_{2 k}(x) h^{2 k}+\mathcal{O}\left(h^{2 K+2}\right)
\end{aligned}
$$

to eliminate the term in $\mathcal{O}\left(h^{2}\right)$. Dividing by $d^{(1)}(h)$ yields (7.1a).

In the induction step, the numerator of the left hand side of (7.1a) yields

$$
\begin{aligned}
& 4^{\ell}\left\{f(x)+\frac{1}{2 \pi} \frac{\sin \left(\frac{\pi}{h / 2} x\right)}{d^{(\ell-1)}(h)} \sum_{k=\ell}^{K}\left[\prod_{i=1}^{\ell-1}\left(4^{i}-4^{k}\right)\right] b_{2 k}(x) h^{2 k}\right\} \\
& -2 \cos \left(\frac{\pi}{h} x\right) \frac{d^{(\ell-1)}(2 h)}{d^{(\ell-1)}(h)} \\
& \cdot\left\{f(x)+\frac{1}{2 \pi} \frac{\sin \left(\frac{\pi}{h} x\right)}{d^{(\ell-1)}(2 h)} \sum_{k=\ell}^{K}\left[\prod_{i=1}^{\ell-1}\left(4^{i}-4^{k}\right)\right] b_{2 k}(x)(2 h)^{2 k}\right\}+\mathcal{O}\left(h^{2 K+2}\right)
\end{aligned}
$$

so that the quotient becomes

$$
\begin{aligned}
C^{(\ell)}\left(\frac{h}{2}\right)= & f(x)+\frac{1}{2 \pi} \frac{1}{d^{(\ell)(h)}} \sum_{k=\ell}^{K}\left[\prod_{i=1}^{\ell-1}\left(4^{i}-4^{k}\right)\right] \\
& \cdot\left(4^{\ell} \sin \left(\frac{\pi}{h / 2} x\right)-2 \cos \left(\frac{\pi}{h} x\right) \sin \left(\frac{\pi}{h} x\right) 4^{k}\right) b_{2 k}(x) h^{2 k}+\mathcal{O}\left(h^{2 K+2}\right),
\end{aligned}
$$

where $d^{(\ell)}(h)$ is defined in (7.1d). It remains to notice that $\sin \left(\frac{\pi}{h / 2} x\right)$ is a common factor and that the term corresponding to $k=\ell$ vanishes to obtain (7.1c).

As with Romberg, but here for every $x$, the values $C^{(\ell)}\left(\frac{h}{2}\right)$ for a given $\frac{h}{2}$ may be collected in the $\ell$ th row of a tableau, which will be triangular since the calculation of $C^{(\ell)}\left(\frac{h}{2}\right)$ requires knowledge of $C^{(\ell-1)}(h)$. For a given $x$ and a given $h$, the elements $T_{k \ell}:=C^{(\ell-1)}\left(\frac{h}{2^{k}}\right)$ of the tableau may be computed by means of the following pseudocode.

$$
\begin{aligned}
& h=X / N ; \\
& T_{11}=C^{(0)}(h)=C_{N}(f, h)(x) ; \\
& g(1)=d^{(1)}(2 h)=4-2(-1)^{N} \cos \left(\frac{\pi}{2 h} x\right) ; \\
& i=1 ; \operatorname{diff}=10^{5} ; \\
& \text { while } i<\operatorname{imax} \& \text { diff }>\text { tol } \\
& \quad i=i+1 ; \\
& \quad \text { hov } 2=h / 2 ; \\
& \quad T_{i 1}=C^{(0)}(\operatorname{hov} 2)=C_{N}(f, \operatorname{hov} 2)(x) ; \\
& \quad a=2 * \cos \left(\frac{\pi}{h} x\right) ;
\end{aligned}
$$




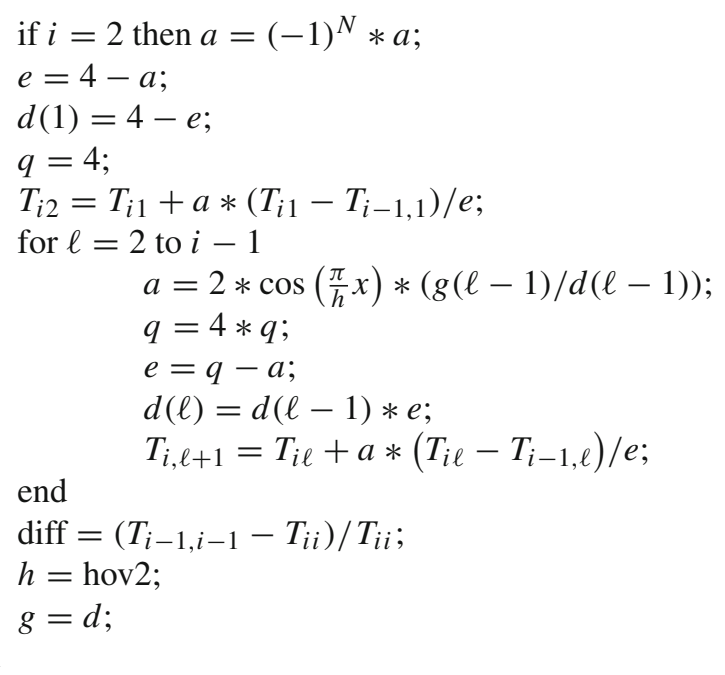

Note that we have written every new approximation as a correction of that on its left, as usual in such tableaux. The if-statement expresses the fact that the factor $(-1)^{N}$ in $a$ is present only for the original value of $h$. The last statement arises from the observation that the $d^{(\ell-1)}(2 h)$ are the denominators of the previous line of the tableau.

In order to experimentally check the order, we have adapted the usual procedure [11, p. 18], to formula (7.1c), in which the polynomial in $h$ is multiplied by the function $\sin \left(\frac{\pi}{h / 2} x\right) / d^{(\ell)}(h)$ that depends on $h$. When the derivatives of $f$ are such that for each entry of the $\ell$ th column the terms of the sum decay, the error in $C^{(\ell)}(h)$ is about

$$
E^{(\ell)}(h):=C^{(\ell)}(h)-f(x) \approx \frac{1}{2 \pi} \frac{\sin \left(\frac{\pi}{h} x\right)}{d^{(\ell)}(2 h)} \prod_{i=1}^{\ell}\left(4^{i}-4^{\ell+1}\right) b_{2 \ell+2}(x) h^{2 \ell+2} .
$$

Dividing this expression by the corresponding one for $\frac{h}{2}$, we obtain

$$
\frac{E^{(\ell)}(h)}{E^{(\ell)}(h / 2)} \approx \frac{\sin \left(\frac{\pi}{h} x\right) / d^{(\ell)}(2 h)}{\sin \left(\frac{\pi}{h / 2} x\right) / d^{(\ell)}(h)} \frac{(2 h)^{2 \ell+2}}{h^{2 \ell+2}}=\frac{1}{2 \cos \left(\frac{\pi}{h} x\right)} \frac{d^{(\ell)}(h)}{d^{(\ell)}(2 h)} 2^{2 \ell+2}
$$

and by taking the base 2 logarithm

$$
2 \ell+2 \approx \log _{2}\left[2 \frac{E^{(\ell)}(h)}{E^{(\ell)}(h / 2)} \cos \left(\frac{\pi}{h} x\right) \frac{d^{(\ell)}(2 h)}{d^{(\ell)}(h)}\right] .
$$

This formula holds for $\ell=0$ too if one sets $d^{(0)}(h)=1 \forall h$.

Example 6 In order to slow down convergence so that the experimental orders of convergence are less subject to cancellation we have taken here $f(x)=\sinh (10 x) /\left(1+x^{2}\right)$, with a small original number of nodes, $2 N+1=9$, still on the interval $[-1,1]$. We 
Table 7 Errors and experimental orders of convergence at $x=\sqrt{2} / 4$ for $f(x)=\sinh (10 x) /\left(1+x^{2}\right)$ on $[-1,1]$ starting from $h=1 / 4$

\begin{tabular}{rrrrrrr}
\hline$\ell=0$ & \multicolumn{1}{l}{1} & \multicolumn{1}{l}{ 2 } & \multicolumn{1}{l}{5} & \multicolumn{1}{l}{6} \\
\hline $3.552 \mathrm{e}+02$ & & & & & \\
$-5.725 \mathrm{e}+01$ & $-8.791 \mathrm{e}+00$ & & & & & \\
$2.604 \mathrm{e}+01$ & $1.033 \mathrm{e}+00$ & $2.635 \mathrm{e}-01$ & & & & \\
$6.256 \mathrm{e}+00$ & $1.257 \mathrm{e}-01$ & $1.278 \mathrm{e}-02$ & $4.022 \mathrm{e}-03$ & & & \\
$-1.735 \mathrm{e}+00$ & $-5.326 \mathrm{e}-03$ & $-1.252 \mathrm{e}-04$ & $-1.241 \mathrm{e}-05$ & $-3.913 \mathrm{e}-06$ & & \\
$3.383 \mathrm{e}-01$ & $2.788 \mathrm{e}-04$ & $1.657 \mathrm{e}-06$ & $4.444 \mathrm{e}-08$ & $4.629 \mathrm{e}-09$ & $1.495 \mathrm{e}-09$ & \\
$-1.178 \mathrm{e}-01$ & $-2.154 \mathrm{e}-05$ & $-3.144 \mathrm{e}-08$ & $-2.138 \mathrm{e}-10$ & $-5.792 \mathrm{e}-12$ & $-3.836 \mathrm{e}-13$ & $5.329 \mathrm{e}-14$ \\
1.7242 & & & & & & \\
1.9158 & 3.8677 & & & & & \\
1.9777 & 2.9589 & 5.1901 & & & & \\
1.9943 & 4.7055 & 6.0757 & 7.5146 & & & \\
1.9986 & 3.8959 & 5.9751 & 7.8466 & 9.4383 & & \\
1.9996 & 4.1719 & 6.0240 & 7.9687 & 9.9034 & 12.187 & \\
\hline
\end{tabular}

used a single evaluation point, first $x=\sqrt{2} / 4$, to keep the results within a two-dimensional array. The results with $i_{\max }=7$ appear in Table 7: the first tableau displays the interpolation errors, the second the experimental orders of convergence. (Since two errors are required for the approximation of an order, the second tableau has one fewer row and column.) The first column shows the $h^{2}$-convergence of the original sinc interpolant (1.1), the next columns the results after the extrapolation steps and the corresponding orders.

With Runge's denominator, $f(x)=\sinh (10 x) /\left(1+25 x^{2}\right)$, the convergence of the Romberg values is even faster (!) but the improvement ceases with an error of about $10^{-11}$ and the orders after 6 are not so nicely evaluated.

When one may increase the size of the sample at will, the difficulty in the vicinity of the boundary mentioned in Sect. 2 does not arise: at $x=\sqrt{0.99} \approx 0.995$ in our last example, starting with three nodes and extrapolating up to $i_{\max }=15$ $(2 N+1=32769)$ gives $f(x)$ with about machine precision and the same experiment with $x=\sqrt{0.999} \approx 0.9995$ still yields a relative accuracy of about $10^{-10}$ with $i_{\max }=17(2 N+1=131073)$. One reason for this precision is the fact that one does not improve upon the sinc interpolant by adding terms containing potentially large derivatives, but rather computes the weighted means of sinc interpolants (which are of the same order of magnitude as $f$ ) given on the left of (7.1c).

The only remaining difficulty at the boundary is Gibbs' phenomenon. As alluded to already, we eliminate it along the lines of [9] by dividing every extrapolated value by the corresponding one for the function 1 . Since the numerator and the denominator have the same behaviour close to the jumps at \pm 1 , not only the phenomenon disappears, but the quotient converges in general faster toward $f(x)$ than the original extrapolated interpolant. That way we introduce quotients of extrapolated sinc interpolants, which 
Table 8 Errors at $x=\sqrt{0.999}$ with quotients of extrapolated sinc interpolants for $f(x)=\sinh (10 x) /(1+$ $\left.x^{2}\right)$

\begin{tabular}{lllllll}
\hline$\ell=0$ & 1 & 2 & 3 & 4 & 5 & 6 \\
\hline $4.0089 \mathrm{e}-03$ & & & & & & \\
$3.3535 \mathrm{e}-03$ & $2.6991 \mathrm{e}-03$ & & & & & \\
$2.3095 \mathrm{e}-03$ & $1.2684 \mathrm{e}-03$ & $1.0645 \mathrm{e}-03$ & & & & \\
$1.3146 \mathrm{e}-03$ & $3.2520 \mathrm{e}-04$ & $1.9109 \mathrm{e}-04$ & $1.6305 \mathrm{e}-04$ & & & \\
$6.8030 \mathrm{e}-04$ & $5.2988 \mathrm{e}-05$ & $1.4470 \mathrm{e}-05$ & $8.8246 \mathrm{e}-06$ & $7.6213 \mathrm{e}-06$ & & \\
$3.3992 \mathrm{e}-04$ & $7.0551 \mathrm{e}-06$ & $6.1782 \mathrm{e}-07$ & $1.7919 \mathrm{e}-07$ & $1.1236 \mathrm{e}-07$ & $9.7930 \mathrm{e}-08$ & \\
$1.6659 \mathrm{e}-04$ & $8.7056 \mathrm{e}-07$ & $2.0493 \mathrm{e}-08$ & $1.9305 \mathrm{e}-09$ & $5.8551 \mathrm{e}-10$ & $3.7472 \mathrm{e}-10$ & $3.2879 \mathrm{e}-10$ \\
\hline
\end{tabular}

we may as well call rational extrapolated sinc interpolants; we shall study them in future work.

Example 7 As displayed in Table 8, about the same relative accuracy of $10^{-10}$ is attained at $x=\sqrt{0.999}$ in the last example with $i_{\max }=7(2 N+1=129)$ instead of $i_{\max }=17$ above, and $i_{\max }=9(2 N+1=513)$ yields machine precision.

\section{One-sided sinc interpolants}

In applications in which the sinc interpolant is used for approximating in the radial direction (see [24] for a recent chemical example), i.e., on the one-sided infinite interval $[0, \infty), f$ will seldom vanish at 0 along with all its derivatives and $f$ extended as zero to the interval $(-\infty, 0)$ will have a jump at the origin. The sinc interpolant converges very slowly even when $f$ decreases very rapidly with growing $x$. If $f$ is analytic, the now classical way of coping with this jump is to conformally map a domain containing the interval $(0, \infty)$ onto a strip about $(-\infty, \infty)$, to use the map to transplant $f$ to this strip, to interpolate the transplanted function and to take the result back to $(0, \infty)[19,21]$. The main disadvantage of this approach is that the map squeezes the interpolation nodes exponentially in the vicinity of 0 which leads, e.g., to ill-conditioned derivatives.

To stick with equidistant nodes, one may use the finite sinc interpolant on an interval $[0, X]$ which we define as $C_{N}(f, h)$ from (1.1) with $f\left(x_{n}\right)=0$ for $n<0$ and $f\left(x_{0}\right):=f\left(x_{0}\right) / 2$, i.e.,

$$
C_{N}(f, h)(x)=\sum_{n=0}^{N} \prime \prime f\left(x_{n}\right) \operatorname{sinc}\left[\frac{\pi}{h}\left(x-x_{n}\right)\right] .
$$

In the case of an odd number of points $N+1=2 L+1$ on the interval $[0, X]$, the formula for $C_{N}(f, h)-f$ is even slightly simpler than (1.3). Indeed $C_{N}$, rolled up on the circle of diameter $\frac{X}{\pi}$ [6], starts and ends at $0 \equiv X$ and this leads to a positive sign 
of the trapezoidal approximations $T_{h}$ of the Cauchy principal value $I_{x}$ underlying $C_{N}$, independently of the parity of $L$ :

$$
C_{N}(f, h)(x)=\frac{1}{2 \pi} \sin \left(\frac{\pi}{h} x\right)\left(T_{2 h}(x)-M_{2 h}(x)\right), \quad I_{x}=\mathrm{PV} \int_{-X}^{X} \frac{f(y)}{x-y} d y
$$

$\left(M_{h}:=\right.$ midpoint rule approximation to $I_{x}$ with the steplength $\left.h[7,8]\right)$. The jump in $f$ on the circle now is at $0 \equiv X$ and the error formula is the same as $(1.3)$ with $(-1)^{M}$ replaced by 1 and $-X$ by 0 as argument of the last derivative.

Also with an even number $N+1=2 L$ of points, the error formula is easier to obtain than in the symmetric case given in [8]. Since one does not have to shift the interval before extending $f$ to an even function interpolated between an odd number of points, the factor of the sum $\sum_{k}$ remains a sine, but with a negative sign since $C_{N}$ is now a difference $M_{2 h}-T_{2 h}$ [8], leading to a negative sign in the error formula too, while the difference of derivatives is again replaced by a sum as in [8], to finally yield the formula

$$
\begin{aligned}
C_{N}(f, h)(x)= & f(x)+\frac{1}{2 \pi} \sin \left(\frac{\pi}{h} x\right) \\
& \cdot 2 \sum_{k=1}^{K}\left(1-4^{-k}\right) \frac{B_{2 k}}{(2 k) !}\left[\left(\frac{f(y)}{x-y}\right)^{(2 k-1)}(X)+\left(\frac{f(y)}{x-y}\right)^{(2 k-1)}\right. \\
& \cdot(2 h)^{2 k}+\mathcal{O}\left(h^{2 K+2}\right) .
\end{aligned}
$$

Table 9 Errors with the quotient of one-sided corrected sinc interpolants for $f(x)=e^{-x^{2}} /\left(1+x^{2}\right)$ on $[0,6]$ with 90 points

\begin{tabular}{lrrrrr}
\hline $\mathrm{x}$ & \multicolumn{1}{l}{1} & \multicolumn{1}{l}{ 2 } & \multicolumn{1}{l}{14} \\
\hline $3.1579 \mathrm{e}-01$ & $-2.9852 \mathrm{e}-03$ & $-1.5699 \mathrm{e}-06$ & $-1.5450 \mathrm{e}-08$ & $-3.0785 \mathrm{e}-10$ & \multicolumn{1}{l}{0} \\
$6.3158 \mathrm{e}-01$ & $8.2943 \mathrm{e}-04$ & $1.3263 \mathrm{e}-06$ & $1.3231 \mathrm{e}-08$ & $2.6452 \mathrm{e}-10$ & $4.4409 \mathrm{e}-16$ \\
$9.4737 \mathrm{e}-01$ & $-6.6463 \mathrm{e}-05$ & $-1.7137 \mathrm{e}-07$ & $-1.7426 \mathrm{e}-09$ & $-3.5007 \mathrm{e}-11$ & $4.1633 \mathrm{e}-16$ \\
$1.2632 \mathrm{e}+00$ & $-1.6729 \mathrm{e}-04$ & $-5.4473 \mathrm{e}-07$ & $-5.6555 \mathrm{e}-09$ & $-1.1423 \mathrm{e}-10$ & $-3.7470 \mathrm{e}-16$ \\
$1.5789 \mathrm{e}+00$ & $1.4115 \mathrm{e}-04$ & $5.1955 \mathrm{e}-07$ & $5.4998 \mathrm{e}-09$ & $1.1168 \mathrm{e}-10$ & 0 \\
$1.8947 \mathrm{e}+00$ & $-3.2841 \mathrm{e}-05$ & $-1.2949 \mathrm{e}-07$ & $-1.3933 \mathrm{e}-09$ & $-2.8428 \mathrm{e}-11$ & $1.7521 \mathrm{e}-16$ \\
$2.2105 \mathrm{e}+00$ & $-4.5649 \mathrm{e}-05$ & $-1.8755 \mathrm{e}-07$ & $-2.0444 \mathrm{e}-09$ & $-4.1881 \mathrm{e}-11$ & $-9.0639 \mathrm{e}-17$ \\
$2.5263 \mathrm{e}+00$ & $5.6714 \mathrm{e}-05$ & $2.3920 \mathrm{e}-07$ & $2.6335 \mathrm{e}-09$ & $5.4127 \mathrm{e}-11$ & $5.5294 \mathrm{e}-18$ \\
$2.8421 \mathrm{e}+00$ & $-2.1402 \mathrm{e}-05$ & $-9.1878 \mathrm{e}-08$ & $-1.0192 \mathrm{e}-09$ & $-2.1004 \mathrm{e}-11$ & $-2.9341 \mathrm{e}-18$ \\
$3.1579 \mathrm{e}+00$ & $-1.7337 \mathrm{e}-05$ & $-7.5358 \mathrm{e}-08$ & $-8.4082 \mathrm{e}-10$ & $-1.7364 \mathrm{e}-11$ & $2.7090 \mathrm{e}-17$ \\
$3.4737 \mathrm{e}+00$ & $3.0002 \mathrm{e}-05$ & $1.3161 \mathrm{e}-07$ & $1.4750 \mathrm{e}-09$ & $3.0512 \mathrm{e}-11$ & $-1.8771 \mathrm{e}-17$ \\
$3.7895 \mathrm{e}+00$ & $-1.5538 \mathrm{e}-05$ & $-6.8630 \mathrm{e}-08$ & $-7.7187 \mathrm{e}-10$ & $-1.5989 \mathrm{e}-11$ & $2.4882 \mathrm{e}-16$ \\
$4.1053 \mathrm{e}+00$ & $-6.9991 \mathrm{e}-06$ & $-3.1080 \mathrm{e}-08$ & $-3.5053 \mathrm{e}-10$ & $-7.2693 \mathrm{e}-12$ & $-1.9863 \mathrm{e}-16$ \\
$4.4211 \mathrm{e}+00$ & $1.8018 \mathrm{e}-05$ & $8.0347 \mathrm{e}-08$ & $9.0820 \mathrm{e}-10$ & $1.8851 \mathrm{e}-11$ & $2.8853 \mathrm{e}-18$ \\
$4.7368 \mathrm{e}+00$ & $-1.1912 \mathrm{e}-05$ & $-5.3300 \mathrm{e}-08$ & $-6.0359 \mathrm{e}-10$ & $-1.2538 \mathrm{e}-11$ & $1.9521 \mathrm{e}-16$ \\
$5.0526 \mathrm{e}+00$ & $-2.3423 \mathrm{e}-06$ & $-1.0509 \mathrm{e}-08$ & $-1.1919 \mathrm{e}-10$ & $-2.4776 \mathrm{e}-12$ & $-1.6693 \mathrm{e}-16$ \\
$5.3684 \mathrm{e}+00$ & $1.1544 \mathrm{e}-05$ & $5.1915 \mathrm{e}-08$ & $5.8953 \mathrm{e}-10$ & $1.2260 \mathrm{e}-11$ & $1.2052 \mathrm{e}-16$ \\
$5.6842 \mathrm{e}+00$ & $-9.4134 \mathrm{e}-06$ & $-4.2410 \mathrm{e}-08$ & $-4.8215 \mathrm{e}-10$ & $-1.0032 \mathrm{e}-11$ & $1.6459 \mathrm{e}-17$ \\
\hline
\end{tabular}


Example 8 We chose an even number of points and interpolated functions which decay rapidly enough to be able to choose $X$ sufficiently large that $(2 h)^{2 k}\left|\left(\frac{f(y)}{x-y}\right)^{(2 k-1)}(X)\right| \leq$ $v$ in (8.1). Table 9 displays the results for $f(x)=e^{-x^{2}} /\left(1+x^{2}\right)$ and $N+1=90$. The various columns have the same meaning as in Table 1.

\section{Conclusion}

The various applications of our formula for the error of the sinc interpolant should have convinced the reader of its usefulness for the infinitely smooth interpolation between equidistant points on the interval. But they also demonstrate that a few things should be taken into account when using it. The interpolant should be made rational, at least when it is to be used in the last half intervals at the extremities. A nice example is the correction of the barycentric formula that permits a perfectly accurate evaluation of the interpolant without locating its argument. When the interpolated values are given as a sample whose size cannot be chosen, so that the correction will be calculated with finite differences, it is better to reserve some extra points on the left and the right of the interval in order to use centered instead of one-sided differences. On the other hand, rational extrapolated sinc interpolants will be a very good choice when one may take the sample size as a large composite number. We intend to study them further in the near future.

Acknowledgments The author thanks the referees for the large number of suggestions that have improved the form of this work. He is sorry for the errors introduced in the course of the revision.

\section{References}

1. Baltensperger, R., Berrut, J.-P.: The linear rational collocation method. J. Comput. Appl. Math. 134, 243-258 (2001)

2. Baltensperger, R., Berrut, J.-P., Noël, B.: Exponential convergence of a linear rational interpolant between transformed Chebyshev points. Math. Comp. 68, 1109-1120 (1999)

3. Bateman Manuscript Project. In: Erdéyi, A., et al. (eds.) Higher Transcendental Functions, vol. I. McGraw-Hill, New York (1953)

4. Berrut, J.-P.: Rational functions for guaranteed and experimentally well-conditioned global interpolation. Comput. Math. Appl. 15, 1-16 (1988)

5. Berrut, J.-P.: Barycentric formulae for cardinal (SINC-) interpolants. Numer. Math. 54, 703-718 (1989); Erratum 55, 747

6. Berrut, J.-P.: A circular interpretation of the Euler-Maclaurin formula. J. Comput. Appl. Math. 189, 375-386 (2006)

7. Berrut, J.-P.: A formula for the error of finite sinc-interpolation over a finite interval. Numer. Algorithms 45, 369-374 (2007)

8. Berrut, J.-P.: A formula for the error of finite sinc-interpolation with an even number of nodes (submitted)

9. Berrut, J.-P.: Fighting Gibbs' phenomenon through quotienting (in preparation)

10. Berrut, J.-P., Trefethen, L.N.: Barycentric Lagrange interpolation. SIAM Rev. 46, 501-517 (2004)

11. de Boor, C.: A Practical Guide to Splines. Revised Edition. Applied Mathematical Sciences, vol. 27, Springer, New York (2001)

12. Floater, M.S., Hormann, K.: Barycentric rational interpolation with no poles and high rates of approximation. Numer. Math. 107, 315-331 (2007) 
13. Fornberg, B.: A Practical Guide to Pseudospectral Methods. Cambridge University Press, Cambridge (1996)

14. Fornberg, B.: Calculation of weights in finite difference formulas. SIAM Rev. 40, 685-691 (1998)

15. Gautschi, W.: Computation of successive derivatives of $f(z) / z$. Math. Comp. 20, 209-214 (1966)

16. Gautschi, W.: Barycentric formulae for cardinal (SINC-)interpolants by Jean-Paul Berrut (Remark). Numer. Math. 87, 791-792 (2001)

17. Håvie, T.: On a modification of Romberg's algorithm. BIT 6, 24-30 (1966)

18. Kress, R.: Interpolation auf einem unendlichen Intervall. Computing 6, 274-288 (1970)

19. Lund, J., Bowers, K.L.: Sinc Methods for Quadrature and Differential Equations. SIAM, Philadelphia (1992)

20. Schwarz, H. R.: Numerische Mathematik $4^{\text {te }}$ Aufl., Teubner, 1997; english translation of the second edition: Numerical Analysis. A Comprehensive Introduction. Wiley, New York (1989)

21. Stenger, F.: Numerical Methods based on Sinc and Analytic Functions. Springer, Heidelberg (1993)

22. Tee, T.W., Trefethen, L.N.: A rational spectral collocation method with adaptively transformed Chebyshev grid points. SIAM J. Sci. Comput. 28, 1798-1811 (2006)

23. de la Vallée Poussin, C. J.: Sur la convergence des formules d'interpolation entre ordonnées équidistantes. Bull. de la Classe des Sci. de l'Acad. Roy. de Belgique. Série 4, 319-410 (1908)

24. Weber, V., Daul, C., Baltensperger, R.: Radial numerical integrations based on the sinc function. Comput. Phys. Commun. 163, 133-142 (2004) 